\title{
Inhalation of Fire Extinguisher Powder
}

\author{
Sigrid Beitland', Olav Stokland', Vidar Skaug ${ }^{2}$, Asbjoern Skogstad², Olav Klingenberg ${ }^{3}$
}

\begin{abstract}
A young man was multitraumatized by a car accident. During the stay in the intensive care unit he had persistent hypoxemia. Various reasons were sought and different treatments were tried, but his severe hypoxemia did not ameliorate. The cause of the hypoxemia remained unclear until his clinical condition improved and we dared to perform a second bronchoscopy which revealed fire extinguisher powder in his airways. The clinical course of the patient is described, as well as the diagnostic and therapeutic measures. Details are given about the methods used for identification of the powder. The problems following unawareness of the condition are discussed.
\end{abstract}

\section{Key Words}

Inhalation injury · Fire extinguisher powder • Multitrauma.ARDS

Eur J Trauma 2006;32:286-291

DOI 10.1007/s00068-006-6052-y

\section{Case Study}

An 18-year-old previously healthy man was the driver of a car that crashed with a bus. The incident happened on a wet and icy road with a speed limit of $80 \mathrm{~km} / \mathrm{h}$. Both vehicles had reduced speed due to difficult driving conditions. There were two passengers in the car and the driver was initially awake and fully responsive. After the accident he was wedged in the car that caught fire. Bystanders tried to stop the flames using snow, water and fire extinguishers from houses nearby and from trucks equipped with fire extinguisher powder that arrived at the scene. The fire fighters quenched the fire after approximately $15 \mathrm{~min}$ using only water. The driver was wedged in the car due to massive deformation of the vehicle for about $55 \mathrm{~min}$. An air ambulance with an anesthesiologist was sent to the accident site, who found him unconscious after the fire with a Glasgow Coma Score of 5 around $20 \mathrm{~min}$ after the incident. He then had a heart rate of 125 beats/min, a blood pressure of $130 / 80 \mathrm{mmHg}$ and a peripheral oxygen saturation of $88 \%$. The minimum body temperature was measured as $35.0^{\circ} \mathrm{C}$. An infusion was started with crystalloid fluid and he was sedated and muscle relaxated before intubation in the field. Using a transport ventilator, he was difficult to ventilate. Volume-controlled ventilation was used with tidal volumes of $700 \mathrm{ml}$; the peak airway pressure was measured to be $40 \mathrm{~cm} \mathrm{H}_{2} \mathrm{O}$. He was given bilateral pleura drainage at the scene due to suspicion of pneumothorax. Arrival at the local hospital was approximately $90 \mathrm{~min}$ after the accident. He was then transferred by air ambulance to our regional trauma centre because of multitrauma, and arrived approximately $7 \mathrm{~h}$ after the accident. He then had stable vital signs with a heart rate of 100 beats/min, a blood pressure of $120 / 50 \mathrm{mmHg}$ and a peripheral oxygen saturation of 99\%. The following injuries were diagnosed:

- Fracture of the transverse process of vertebrae $\mathrm{C} 7$ and TH1.

- Fracture of the clavicle left side.

- Fracture of costae 1-5 left side.

- Bilateral lung contusions and pneumothorax.

- Spleen injury grade 1.

- Fracture of the distal humerus left side.

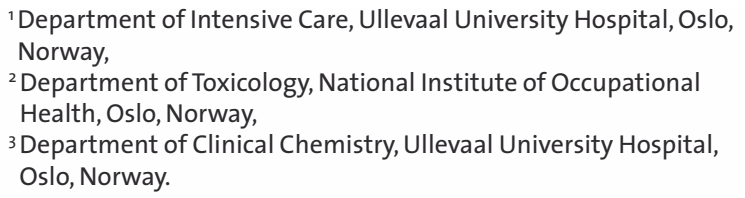

Received: May 2, 2005; revision accepted: December 30, 2005. 
- Bilateral cruz fractures.

- Mucocutaneous injuries on the left knee and leg.

- Third grade burn injuries on both hands and knees (approximately $5 \%$ of total body surface area).

He underwent a total of 16 orthopedic and 25 plastic surgical procedures during the hospital stay in addition to a tracheotomy; some had to be postponed due to hypoxemia. A total of seven pleura drains were used on the right and four pleura drains on the left side, all because of pneumothorax. No additional thoracic surgery was performed. He was circulatory stable during ICU stay, except for periods of bleeding and infections. He had a sinus rhythm with a heart rate varying from 50 to 140 beats/min, averaging at about 120 beats/min. The possibility of a circulatory failure causing hypoxemia was investigated. Transthoracic echocardiography was preformed three times, the first to access cardiac function and exclude the presence of an anatomic shunt. The next two investigations were done during infections to optimize volume status and vasoactive medications. There was no presence of atrial or ventricular septum defects causing shunting of blood. The anatomic structure of the heart was normal, with no valvular or myocardial abnormalities. During periods of infections the circulatory system was further monitored using a pulmonary artery catheter and later a pulse contour cardiac output examination. The measurements confirmed the hyperdynamic circulation with a cardiac index of 4.6-7.8 $1 / \mathrm{min} / \mathrm{m}^{2}$. The systemic vascular resistance index was normal to low (560-1,900 dynes $\left.\mathrm{s} / \mathrm{cm}^{5} / \mathrm{m}^{2}\right)$, and the pulmonary vascular resistance index was normal to high (250-450 dynes $\mathrm{s} / \mathrm{cm}^{5} / \mathrm{m}^{2}$ ). Central venous oxygen saturation $(\mathrm{SvO} 2)$ was measured intermittently, with a minimum value of $54 \%$. The volume status was optimized using central filling pressures and echocardiography. Dobutamin infusion in doses of $1-8 \mu \mathrm{g} / \mathrm{kg} / \mathrm{min}$ was used for a total of 26 days to increase cardiac output and thereby the oxygen carrying capacity of blood. During reduction of dobutamin infusion rate, a lowering in cardiac output and worsening of hypoxemia were noted. Norepinephrine in doses of $0.01-0.18 \mu \mathrm{g} / \mathrm{kg} / \mathrm{min}$ was used for a total of 4 days during infections causing low systemic vascular resistance and systemic hypotension. The aim of the treatment was to keep a sufficient tissue oxygenation and the mean arterial pressure above $60 \mathrm{mmHg}$, being enough to keep a normal urine output. He had a sustained critical oxygenation and ventilation failure with recurrent bilateral pneumothorax and bacterial pneumonias. The bacterial agents causing pneumo- nia (and the antibiotic treatment) were the following: Staphylococcus aureus (clindamycin), Haemophilus influenzea (ciprofloxacin), Klebsiella oxytoca (piperacillin/tazobactam) and Stenotrophomonas maltophilia (trimetoprim-sulpha). In addition, Candida albicans was treated with fluconazole. During pleura drainage insertion, the presence of pleural adhesions was problematic. Bronchial secretion was moderate, and no significant bronchospasm was noted. Chest X-rays and CT scans showed diffuse bilateral lung infiltrates. Oxygenation was the major problem causing lactic acidosis and peripheral oxygen saturation down to $65 \%$, ventilation was less problematic. The fraction of inspired oxygen was above 0.5 for the first 45 days, with a minimal arterial oxygen tension of $6.2 \mathrm{kPa}$. The hypoxemia was so severe that the ARDS-criteria $\left(\mathrm{PaO}_{2} / \mathrm{FiO}_{2}\right.$-ratio $\left.<26.67 \mathrm{kPa}\right)$ were fulfilled for 62 consecutive days (Figure 1) [1]. The minute ventilation was between 6.2 and $18.2 \mathrm{l} / \mathrm{min}$, with a maximum arterial carbon dioxide tension of $11.8 \mathrm{kPa}$. The maximal peak airway pressure measured on the ventilator was $38 \mathrm{~cm} \mathrm{H}_{2} \mathrm{O}$.

Inhalation injury was not suspected because the initial bronchoscopy did not reveal soot in his airways, and the carbon monoxide level in blood was normal. His oxygenation problem was treated with biphasic positive pressure ventilation. Low tidal volume ventilation and permissive hypercapnia was used. An elevated oxygen carrying capacity in the blood was kept in periods of hypoxemia. High hemoglobin levels (> $11 \mathrm{~g} / \mathrm{dl}$ ) were used. His oxygen demand was reduced by lowering the body temperature (to a minimum of $34.5^{\circ} \mathrm{C}$ ) using an intravascular cooling device. Even though it is not well documented, it did have a positive effect on his oxygenation. He had persistent hypoxemia and was

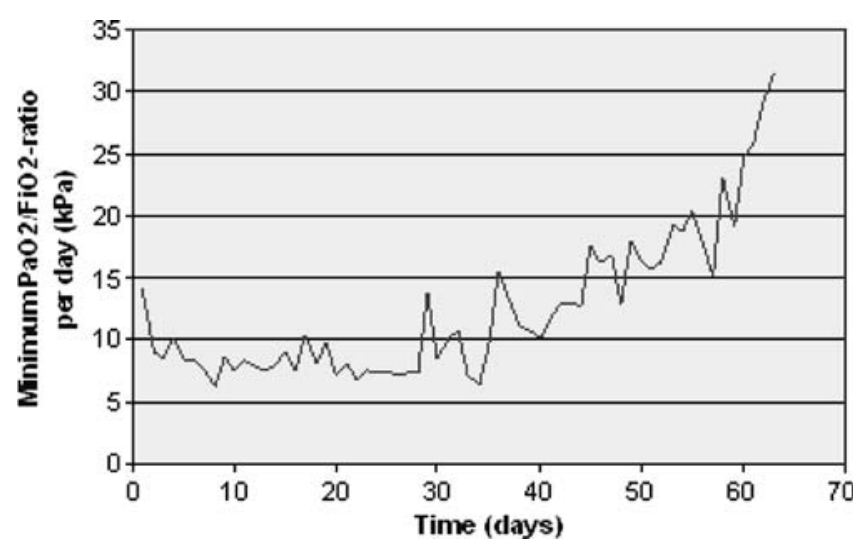

Figure 1. Graphic presentation of the patient's minimum $\mathrm{PaO}_{2} /$ $\mathrm{FiO}_{2}$-ratio per day. 
given high doses of methylprednisolon $(500 \mathrm{mg} \times 3$ for 3 days), without any improvement. This treatment is not evidence based and was given due to severe ARDS for several weeks with lack of amelioration. The treatment was stopped because no positive response on oxygenation was observed. Several other therapeutic options were considered, but not used. The condition was life threatening for several weeks due to infections and failure of the circulatory, respiratory, renal and coagulation systems. He had moderate amounts of bronchial secretion and no lobar atelectasis. During uncoupling of the ventilator circuit his oxygenation worsened, therefore a closed suction system was used. After 32 days his hypoxemia was less severe, so a bronchoscopy could be performed. The indication was need of bacterial samples, and it was then considered safe because of improvement of lung function. The bronchoscopy showed a massive accumulation of white material in his trachea and bronchi (Figure 2). Suspecting the material to be fire extinguisher powder, a broncheoalveolar lavage was done and samples sent for chemical analysis.

The patient got standard intensive care treatment. After 2 months he was weaned from the ventilator and his lung function improved rapidly.

\section{Verification of Fire Extinguisher Powder Background}

The white material in the patients' airways was suspected to be fire extinguisher powder. Information from the firemen at the scene confirmed the use of both $\mathrm{ABC}$ and BC-powder. These kinds of powders are widely used internationally. The Norwegian Fire Research Laboratory was contacted, and information about the chemical content was the basis for analysis.

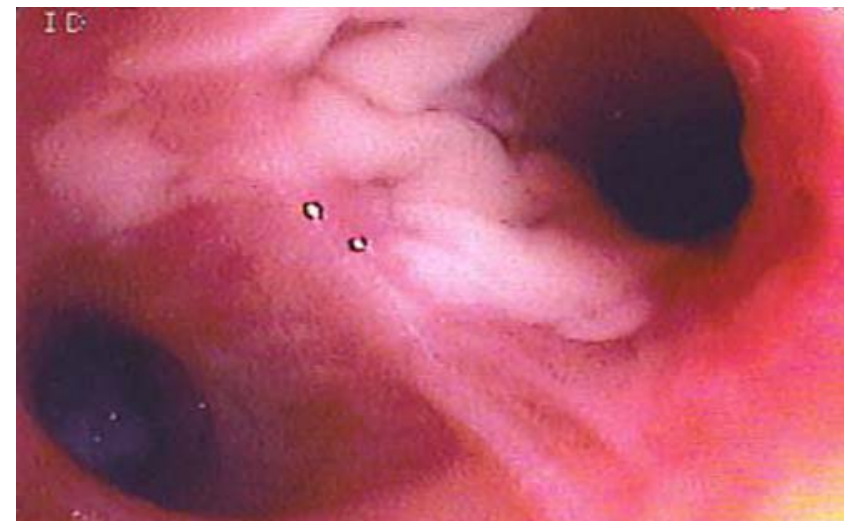

Figure 2. Bronchoscopic picture of the white material in the trachea and bronchi.

\section{Methods}

The presence of ammonium, carbonate and phosphate in the broncheoalveolar lavage fluid was checked by standard qualitative chemical analysis. Samples of commercial $\mathrm{ABC}$ and $\mathrm{BC}$ fire extinguisher powder were chemically compared with mineral particles in the patients' broncheoalveolar lavage using scanning electron microscopy and energy dispersive X-ray spectroscopy. The samples of ABC- and BC-powder were made airborne in a small dust chamber by introducing jets of compressed air and then allowed to settle on sticky carbon tabs. The tabs were mounted on aluminium stubs and coated with carbon in a Balzers SCD 050 sputter coater (Balzers, Liechtenstein). The broncheoalveolar lavage liquid was diluted and filtered on to a polycarbonate filter and prepared for analytic scanning electron microscopy examination. Particle counting and characterization were preformed automatically in a Jeol JSM-6400 scanning electron microscope (Akishima, Tokyo, Japan) connected to a Vantage DSI energy dispersive X-ray spectrometer (Thermo NORAN, Middleton, WI, USA) equipped with a thin window (NORVAR) detector, which allows for detection of elements with an atomic number $\geq 5$. About 80 particles from each sample were characterized.

\section{Results}

The broncheoalveolar lavage material was analysed by standard qualitative chemical analysis and showed presence of ammonium, carbonate and phosphate. The analytical scanning electron microscopy demonstrated large amounts of particles in fluid ranging from less than 1 to $50 \mu \mathrm{m}$ in diameter. The particle surfaces were rough with no signs of cracking or aggregation. The chemical spectra found by energy dispersive X-ray spectroscopy revealed that calcium was the major element in the largest particles. Other identified elements were oxygen, carbon, phosphorous and sodium, as well as sulphur and potassium. Very few particles were identified as quartz particles. We concluded that the chemical content of the broncheoalveolar lavage fluid was similar to the commercial powders, as shown in Figures 3, 4, and 5.

\section{Discussion}

Inhalation injury in fire victims is well described in the literature focusing on inhalation of soot, carbon monoxide, hydrogen cyanide and irritating compounds [2-4]. The clinicians are therefore well versed with the diagnosis, treatment and prognosis of these conditions. 

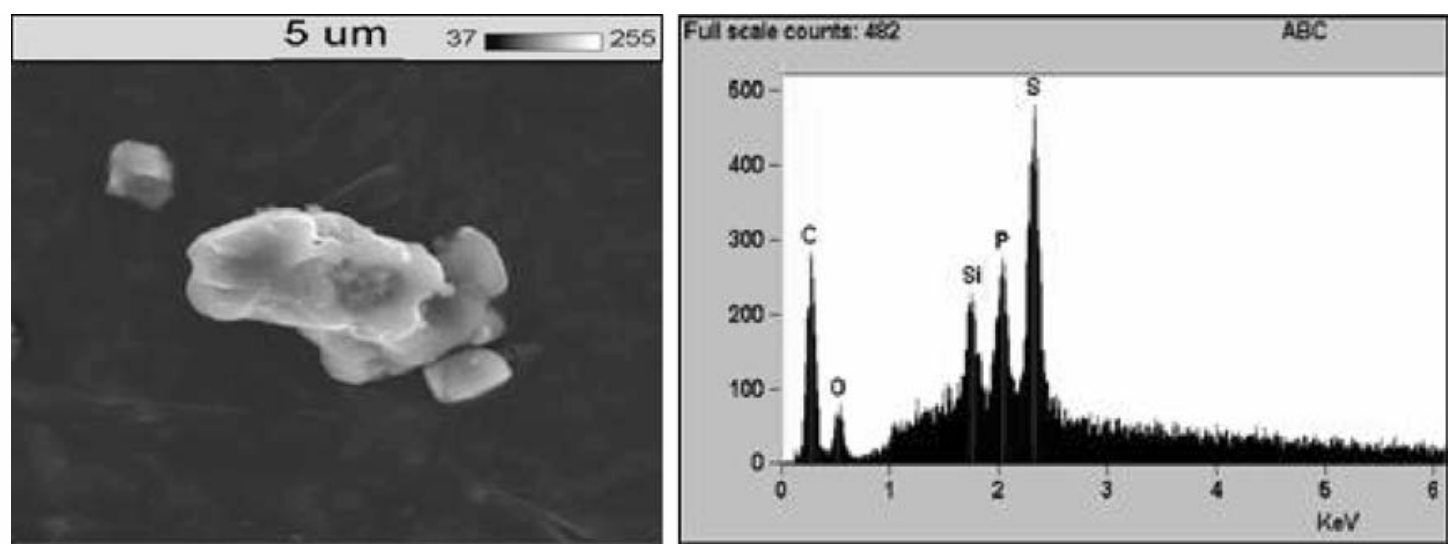

Figure 3. Scanning electron microscopy (left) and energy dispersive X-ray spectroscopy (right) of the ABC fire extinguisher powder. The chemical spectrum shows mainly sulphur (S), carbon (C) and phosphorus (P).
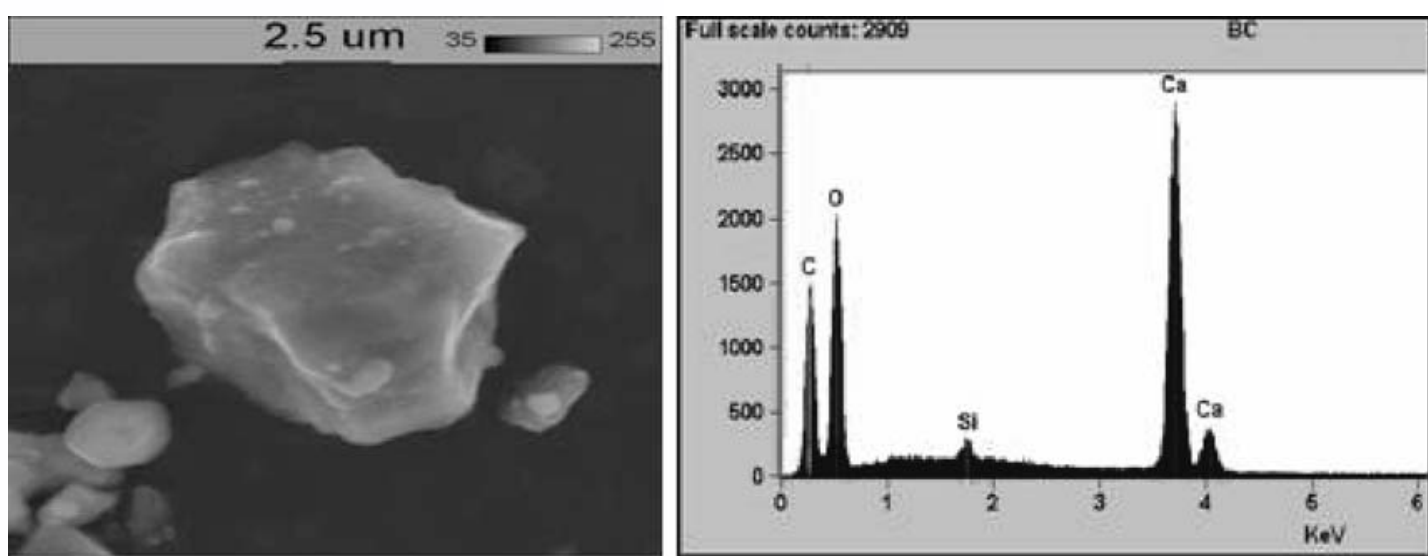

Figure 4. Scanning electron microscopy (left) and energy dispersive X-ray spectroscopy (right) of the BC fire extinguishe powder. The chemical spectrum shows mainly calcium (Ca), oxygen (O) and carbon (C).
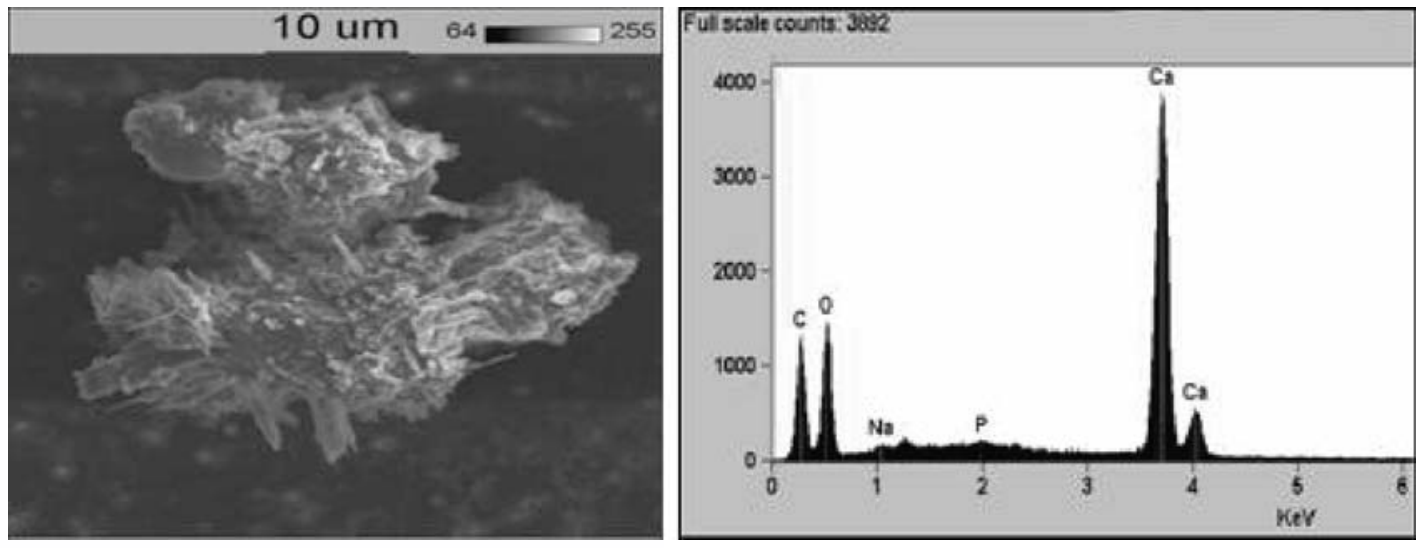

Figure 5. Scanning electron microscopy (left) and energy dispersive X-ray spectroscopy (right) of the patient's broncheoalveolar lavage material. The chemical spectrum shows mainly calcium (Ca), oxygen (O) and carbon (C).

In contrast, acute reactions after inhalation of currently used ABC- or BC-powder are not well known. We found only two published human cases in autopsy materials $[5,6]$. In these cases white powder was found in the airways on post-mortem and the composition was the same as that of the fire extinguisher material. We found similar material in the broncheoalveolar lavage fluid in our patient. The fact that our patient was young and 
athletic may have contributed to his survival, taking into account that his premorbid lung function probably was very good. Hyperphosphatemia causing cardiac arrest has been described after inhalation of dry chemical extinguisher [7]. Our patient's serum levels of calcium, phosphorous and sodium were retrospectively checked and found within physiological limits. Inhalation of fluorocarbons from old fire extinguishers has been studied [8]. The undesirable effects were mainly neurological, cardiovascular and respiratory, the latter dominated by bronchial hyperreactivity. Use of these devices is now limited [9]. Adverse effects on lung function in fire fighters [10] and prolonged effects after smoke inhalation [11] have been investigated in clinical trials. These focus upon long-time reduction of lung function and inflammatory reactions. Articles on adverse effects of the fire extinguisher powder in animals present studies on allergic properties [12]. In our patient no allergic reactions such as rash, excessive bronchial secretion or bronchospasm were noted. Formation of airway particles of non-respiratory diameters in the lungs after inhalation has previously been illustrated [13]. Therefore, it is not surprising that particles up to $50 \mu \mathrm{m}$ in diameter were found in the broncheoalveolar lavage in our patient.

The first bronchoscopy was done by a lung specialist due to a possible inhalation injury. This bronchoscopy revealed no signs of fire extinguisher powder in the airways. The bronchial secretion after this time was moderate. Bronchial suction was performed repeatedly, but no signs of foreign material were revealed. Further bronchoscopy was considered in collaboration with experienced lung specialists. However, his peripheral oxygen saturation fell to about $70 \%$ during uncoupling of the airway system despite preoxygenation, and enhancement of oxygen saturation was thereafter difficult. The potential benefit of a bronchoscopy was considered limited due to lack of major atelectasis and bronchial secretions. We found bronchoscopy too hazardous until day 32 when his lung function did ameliorate. During this bronchoscopy all the visible material was removed using broncheoalveolar lavage. It took about 2 weeks to get chemical confirmation of the presence of fire extinguisher powder. After these 2 weeks his lung function was improving, and further bronchoscopies were considered unnecessary. His severe ARDS was initially found compatible with complications after multitrauma and pneumonia [1416]. Multitrauma may have contributed to his lung injury. However, his oxygenation failure lasted very long, which in our trauma centre is very unusual. The presence of fire extinguisher powder cannot be ruled out as an explanation for his ARDS. We had no suspicion of lung inflammation due to inhalation of foreign materials until the second bronchoscopy could be performed at an acceptable risk on day 32. This material was not described during the first bronchoscopy, maybe because the lung specialist was looking for soot and sings of gastric aspiration. The final diagnosis was dependent upon help from experts in inhalation toxicology and clinical chemistry. By comparing the chemicalcomposition and scanningelectronmicrographs of bulk materials and broncheoalveolar lavage fluid, we found that the patient had inhaled fire extinguisher powder. Retrospectively, we should have been more aware of the use of fire extinguisher powder. However, the lack of classical inhalation injury delayed the diagnosis. After the diagnosis of inhalation injury was made, further options for removal of the material from the lungs were discussed. Multiple broncheoalveolar lavages and application of possible solving chemicals to his airways were not done since his lung function was improving. If the aspiration of fire extinguishing powder was detected earlier, repeated broncheoalveolar lavage probably would have been done. It was also considered experimental and possibly damaging, since we did not find any support for specific treatment in the literature. During the treatment period, the lack of knowledge made it difficult to know how best to treat the patient and what prognosis to expect. Since search in medical databases gave no answers to these questions, we find that this case report may be of value.

\section{Conclusion}

Among various reasons for severe ARDS in multitraumatized patients, one should be aware of possible lung injuries caused by inhalation of standard fire extinguisher powders. The clinicians should think of this possibility, especially when white material is detected in fire victims' airways. The best treatment is probably early and repeated broncheoalveolar lavages, in addition to standard supportive care.

\section{References}

1. Bernard GR, Artigas A, Bringham KL, et al. Report of the American-European consensus conference on ARDS: definitions, mechanisms, relevant outcomes, and clinical trial coordination. Intensive Care Med 1994;20:225-32.

2. Alarie Y. Toxicity of fire smoke. Crit Rev Toxicol 2002;32:259-89.

3. Dries D. More than smoke with fire. Crit Care Med 2002;30:2159-6o. 
4. Trabor DL, Herndon DN, Soejima K. The pathophysiology of inhalation injury. In: Total burn care, 2nd edn. London: Saunders, 2002;221-31.

5. Suzuki T, Takahashi K, Umetsu K. Unusual aspiration in fire death. Forensic Sci Int 1995;72:71-6.

6. Dirnhofer R, Sigrist T. Exceptional suicide of a physician. Z Rechtsmed 1978; 27:81:227-35.

7. Doyon S, McGrath JM. Hyperphosphatemia and cardiac arrest following inhalation of a dry chemical extinguisher. Abstracts. J Toxicol Clin Toxicol 2003;41:609-40.

8. Matrat M, Laurence MF, Iwatsubo Y, et al. Reactive airways dysfunction syndrome caused by bromochlorodifluoromethane from fire extinguishers. Occup Environ Med 2004;61:712-4.

9. Regulation (EC) No. 2037/2000 of the European Parlament and of the Council of 29 June 2000 on substances that deplete the ozone layer. Official Journal of the European Community, 29 September 2000:L244/1-L224/24

10. Brandt-Rauf PW, Fallon LF, Tarantini T, et al. Health hazards of fire fighters: exposure assessment. Br J Ind Med 1988;45:606-12.

11. Park GY, Park JW, Jeong DH, et al. Prolonged airway and systemic inflammatory reactions after smoke inhalation. Chest 2003;123:475-80.

12. Hoymann HG, et al. Evaluation of the respiratory allergenicity of a fire-extinguisher powder in a rat model of allergic asthma. In: International inhalation symposium, Hannover, vol 6, 1997.
13. Hayashi K, et al. Electron microscopic studies on pulmonary alveolar proteinose with interstitial fibrosis caused by inhalation of fire extinguisher powder. J Clin Electron MicrosC 1979;12:913-4.

14. Vincent JL, Akca S, de Mendonca A, et al. The epidemiology of acute respiratory failure in critically ill patients. Chest 2002;121:1602-9.

15. Ware LB, Matthay MA. The acute respiratory distress syndrome. N Engl J Med 2000;342:1334-47.

16. Pelosi P, Gattinoni L. Acute respiratory distress syndrome of pulmonary and extra-pulmonary origin: fancy or reality? Intensive Care Med 2001;27:457-6o.

\section{Address for Correspondence}

Sigrid Beitland, MD

Department of Intensive Care

Ullevaal University Hospital

Oslo, Norway

Phone: (+47/230) 5446

e-mail: sigrid.beitland@ulleval.no 